\title{
Cosmic-Ray Lithium and Beryllium Isotopes in the PAMELA-Experiment
}

\author{
W. Menn ${ }^{* 18}$, E. A. Bogomolov ${ }^{11}$, M. Simon ${ }^{18}$, G. I. Vasilyev $^{11}$, O. Adriani ${ }^{4,5}$, \\ G. C. Barbarino ${ }^{6,7}$, G. A. Bazilevskaya ${ }^{8}$, R. Bellotti ${ }^{9,10}$, M. Boezio ${ }^{1}$, M. Bongi ${ }^{3,5}$, \\ V. Bonvicini ${ }^{1}$, S. Bottai ${ }^{5}$, A. Bruno ${ }^{9,10}$, F. Cafagna ${ }^{10}$, D. Campana ${ }^{7}$, P. Carlson ${ }^{12}$, \\ M. Casolino ${ }^{2,14}$, G. Castellini ${ }^{15}$, C. De Donato ${ }^{2}$, C. De Santis ${ }^{13}$, V. di Felice ${ }^{2,3}$, \\ A. M. Galper ${ }^{16}$, A. V. Karelin ${ }^{16}$, S. V. Koldashov ${ }^{16}$, S. Koldobskiy ${ }^{16}$, S. Y. Krutkov ${ }^{11}$, \\ A. N. Kvashnin ${ }^{8}$, A. Leonov ${ }^{16}$,V. Malakhov ${ }^{16}$, L. Marcelli ${ }^{13}$, M. Martucci ${ }^{13,17}$, \\ A. G. Mayorov ${ }^{16}$, M. Merge ${ }^{2,13}$, V. V. Mikhailov ${ }^{16}$,E. Mocchiutti ${ }^{1}$, A. Monaco ${ }^{9,10}$, \\ N. Mori ${ }^{5}$, R. Munini ${ }^{1}$, G. Osteria ${ }^{7}$, B. Panico ${ }^{7}$ P. Papini ${ }^{5}$, M. Pearce ${ }^{12}$, P. Picozza $^{2,13}$, \\ M. Ricci ${ }^{17}$, S. B. Ricciarini ${ }^{15}$, R. Sparvoli ${ }^{2,13}$, P. Spillantini ${ }^{4,5}$, Y. I. Stozhkov ${ }^{8}$, \\ A. Vacchi ${ }^{1,20}$, E. Vannuccini ${ }^{5}$, S. A. Voronov ${ }^{16}$, Y. T. Yurkin ${ }^{16}$, G. Zampa $^{1}$, N. Zampa ${ }^{1}$, \\ ${ }^{1}$ INFN, Sezione di Trieste I-34149 Trieste, Italy \\ 2 INFN, Sezione di Rome "Tor Vergata", I-00133 Rome, Italy \\ ${ }^{3}$ Agenzia Spaziale Italiana (ASI) Science Data Center, I-00044 Frascati, Italy \\ ${ }^{4}$ University of Florence, Department of Physics, I-50019 Sesto Fiorentino, Florence, Italy \\ ${ }^{5}$ INFN, Sezione di Florence, I-50019 Sesto Fiorentino, Florence, Italy \\ ${ }^{6}$ University of Naples "Federico II", Department of Physics, I-80126 Naples, Italy \\ ${ }^{7}$ INFN, Sezione di Naples, I-80126 Naples, Italy \\ ${ }^{8}$ Lebedev Physical Institute, RU-119991, Moscow, Russia \\ ${ }^{9}$ University of Bari, Department of Physics, I-70126 Bari, Italy \\ ${ }^{10}$ INFN, Sezione di Bari, I-70126 Bari, Italy \\ ${ }^{11}$ Ioffe Physical Technical Institute, RU-194021 St. Petersburg, Russia \\ ${ }^{12}$ KTH, Department of Physics, and the Oskar Klein Centre for Cosmoparticle Physics, AlbaNova \\ University Centre, SE-10691 Stockholm, Sweden \\ ${ }^{13}$ University of Rome "Tor Vergata", Department of Physics, I-00133 Rome, Italy \\ ${ }^{14}$ RIKEN, Advanced Science Institute, Wako-shi, Saitama, Japan \\ ${ }^{15}$ IFAC, I-50019 Sesto Fiorentino, Florence, Italy \\ ${ }^{16}$ National Research Nuclear University MEPhI, RU-115409 Moscow \\ ${ }_{17}$ INFN, Laboratori Nazionali di Frascati, Via Enrico Fermi 40, I-00044 Frascati, Italy \\ ${ }^{18}$ Universität Siegen, Department of Physics, D-57068 Siegen, Germany \\ ${ }^{19}$ INFN, Sezione di Perugia, I-06123 Perugia, Italy \\ ${ }^{20}$ University of Udine, Department of Mathematics and Informatics, I-33100 Udine, Italy
}

E-mail: mennepamela.physik.uni-siegen.de 
The PAMELA space experiment was launched on the 15th of June 2006 from the Baikonur cosmodrome. The scientific objectives addressed by the mission are the measurement of the antiprotons and positrons spectra in cosmic rays, the hunt for antinuclei as well as the determination of light nuclei fluxes from hydrogen to oxygen in a wide energy range and with high statistics. The apparatus comprises a time-of-flight system, a magnetic spectrometer (permanent magnet) with an silicon-microstrip tracking system, an imaging calorimeter built from layers of siliconmicrostrip detectors interleaved with plates of tungsten, an anti-coincidence system, a shower tail scintillator-counter and a neutron detector. The instrument in its detector-combination is also capable to identify isotopes, using the rigidity information from the magnetic spectrometer together with the time-of-flight measurement or with the multiple $\mathrm{dE} / \mathrm{dx}$ measurement in the calorimeter. In this paper details about the analysis method and new results of the isotopic ratios of lithium and beryllium with increased statistics will be presented.

35th International Cosmic Ray Conference - ICRC2017

10-20 July, 2017

Bexco, Busan, Korea

* Speaker. 


\section{Introduction}

The "secondary" constituents of the cosmic rays, e.g. lithium, beryllium, and boron, are produced by spallation in interactions of heavier nuclei, mainly carbon, nitrogen, and oxygen, with the ambient gas in our galaxy. The ${ }^{6} \mathrm{Li}$ isotope is a pure product of interactions of galactic cosmic rays (GCR) with the interstellar medium (ISM). ${ }^{7} \mathrm{Li}$ will be produced in the same way, but stellar production and primordial nucleosynthesis are also possible sources. All measurements of the ${ }^{7} \mathrm{Li} /$ ${ }^{6} \mathrm{Li}$ ratio in the past have values which are much smaller than the value $\sim 12$ determined from meteorites [5] or the value of $12.5 \pm 4.0$ if a stellar contribution to the interstellar medium is regarded [目]. The main contribution to Be isotopes comes from spallation reactions between heavier cosmic rays and the ISM. ${ }^{7} \mathrm{Be}$ decays only by electron capture (half-life on earth $=53$ days), thus the half live in space depends on the electron density and the galactic cosmic ray lifetime. ${ }^{9} \mathrm{Be}$ is stable, but ${ }^{10} \mathrm{Be}$ has a half-life of $1.5 \times 10^{6}$ years years, which is comparable to the characteristic storage time expected for the galactic containment. Therefore measurements of the elemental and isotopic composition of these cosmic rays is important to constrain production and propagation models.

In this paper we present results of the PAMELA satellite experiment. PAMELA has been put in a polar elliptical orbit at an altitude between $\sim 350$ and $\sim 600 \mathrm{~km}$ with an inclination of $70^{\circ}$ as part of the Russian Resurs-DK1 spacecraft. For this analysis we use data gathered between July 2006 and September 2014, about 60000 lithium nuclei and about 32000 beryllium nuclei were selected in the energy interval between 100 and $2000 \mathrm{MeV} / \mathrm{n}$.

\section{The PAMELA instrument}

The PAMELA satellite-borne cosmic ray experiment was built to measure charged particles in the cosmic radiation with a particular focus on antiparticles. It was mounted on the Resurs DK1 satellite and launched from the Baikonur cosmodrome on June 15th 2006. The PAMELA apparatus is composed of several sub-detectors: Time-of-Flight (ToF) system, anti-coincidence system (CARD, CAS, CAT), magnetic spectrometer with microstrip silicon tracking system, W/Si electromagnetic imaging calorimeter, shower-tail-catcher scintillator (S4) and neutron detector. A detailed description of the PAMELA instrument and an overview of the mission can be found in [ [ $]$ ]. The core of the instrument is a magnetic spectrometer, made of a permanent magnet $(0.43 \mathrm{~T})$ and a silicon tracking system (resolution in the bending side $4 \mu \mathrm{m}$ ) for a maximum detectable rigidity of $1 \mathrm{TV}$. The ToF system is divided in 6 layers, arranged in three planes, each plane composed of two layers. The first plane (S1) is placed on top of the instrument, the second plane (S2) is placed between the TRD and the spectrometer, the last plane (S3) is placed between the spectrometer and the calorimeter, just below the magnet. The overall time resolution of the ToF system is about 250 ps for $Z=1$ particles, about 100 ps for $Z=2$ particles [3]], about 85 ps for $Z=3$ particles and about 80 ps for $Z=4$ particles. The W/Si sampling imaging calorimeter comprises 44 single-sided silicon strip detector planes interleaved with 22 plates of tungsten absorber [ [ $]$ ]. Each tungsten layer has a thickness of 0.74 radiation lengths $(2.6 \mathrm{~mm})$ and it is sandwiched between two printed circuit boards, which house the silicon detectors as well as the frontend and digitizing electronics. Each silicon plane consists of $3 \times 3,380 \mu \mathrm{m}$ thick, $8 \times 8 \mathrm{~cm}^{2}$ detectors, segmented into 32 strips with a pitch of $2.4 \mathrm{~mm}$. The orientation of the strips for two consecutive silicon planes is shifted by 90 
degrees, thus providing 2-dimensional spatial information. The total depth of the calorimeter is 16.3 radiation lengths and 0.6 nuclear interaction lengths.

\section{Data analysis}

\subsection{Event selection}

The selection criteria applied to each triggered event will select positively charged particles with a precise measurement of the absolute value of the particle rigidity and velocity. The analysis procedure was similar to the previous work on the hydrogen and helium isotopes [3]. For the analysis of $|Z|>2$ particles a modified position finding algorithm has been developed, which is used in this analysis. For more details of the algorithm see []. Selected tracks must have at least 3 hits on the $x$-view and at least 2 hits on the $y$-view, for the $x$-view we require a "lever arm" (distance between the lowermost and uppermost layer) $\geq 4$. These selection criteria are less strict then the ones used in the work on the hydrogen and helium isotopes (at least 4 hits on the $x$-view and at least 3 hits on the $y$-view) [B] to increase the statistics. We used the full Monte Carlo simulation of the PAMELA apparatus based on the GEANT 4 code [四], which has been already described in [四], to check how the momentum resolution increased when using these less strict criteria. In the rigidity range $1-5 \mathrm{GV}$, which is used for the analysis, the effect is practically negligible. Galactic events were selected if the particles rigidity exceeded the critical rigidity, $\rho_{c}$, defined as 1.3 times the cutoff rigidity $\rho_{S V C}$ computed in the Störmer vertical approximation. Lithium and beryllium events have been selected by means of ionization energy losses in the TOF system. Charge consistency has been required between $\mathrm{S} 12$ and $<\mathrm{S} 2>$ and $<\mathrm{S} 3>$ (the arithmetic mean of the ionizations for the two layers constituting S2 and S3, respectively). Requiring charge consistency above and below the tracking system rejected events interacting in the silicon layers [[]]. A positive value for the measured time of flight ensures that the particle enters PAMELA from above.

\subsection{Isotope separation in the PAMELA instrument}

In each sample of $Z=3$ and $Z=4$ particles an isotopic separation at fixed rigidity is possible since the mass of each particle follows the relation $m=R Z e / \gamma \beta c$ ( $R$ is the magnetic rigidity, $Z \times e$ is the particle charge, and $\gamma$ is the Lorentz factor). The particle velocity $\beta$ can either be provided directly from the timing measurement of the ToF system, or indirectly from the energy loss in the calorimeter, which follows $\beta$ via the Bethe-Bloch formula $d E / d x \propto Z^{2} / \beta^{2}$ (neglecting logarithmic terms).

For the ToF analysis we can use directly the $\beta$ provided by the timing measurement. In Fig. $\square$ we show $\beta$ vs. the particle rigidity for $Z=3$ and $Z=4$ data. The black lines in the figure represent the expectations for each isotope.

The isotopic analysis of nuclei with the calorimeter is restricted to events which do not interact inside the calorimeter. To check if an interaction occurs, we selected events by applying cuts on the ratio $\Sigma q_{\text {track }} / \Sigma q_{\text {tot }}$ in each layer, where $q_{\text {track }}$ is the energy deposited in the strip closest to the track and the neighboring strip on each side, and $q_{t o t}$ the total energy detected in that layer. In case of no interaction, the fraction of $q_{\text {track }} / q_{\text {tot }}$ will be equal to one, if strips outside the track are hit, the value is less then one. The selection is described in more detail in [B]. In a single silicon layer, the energy 

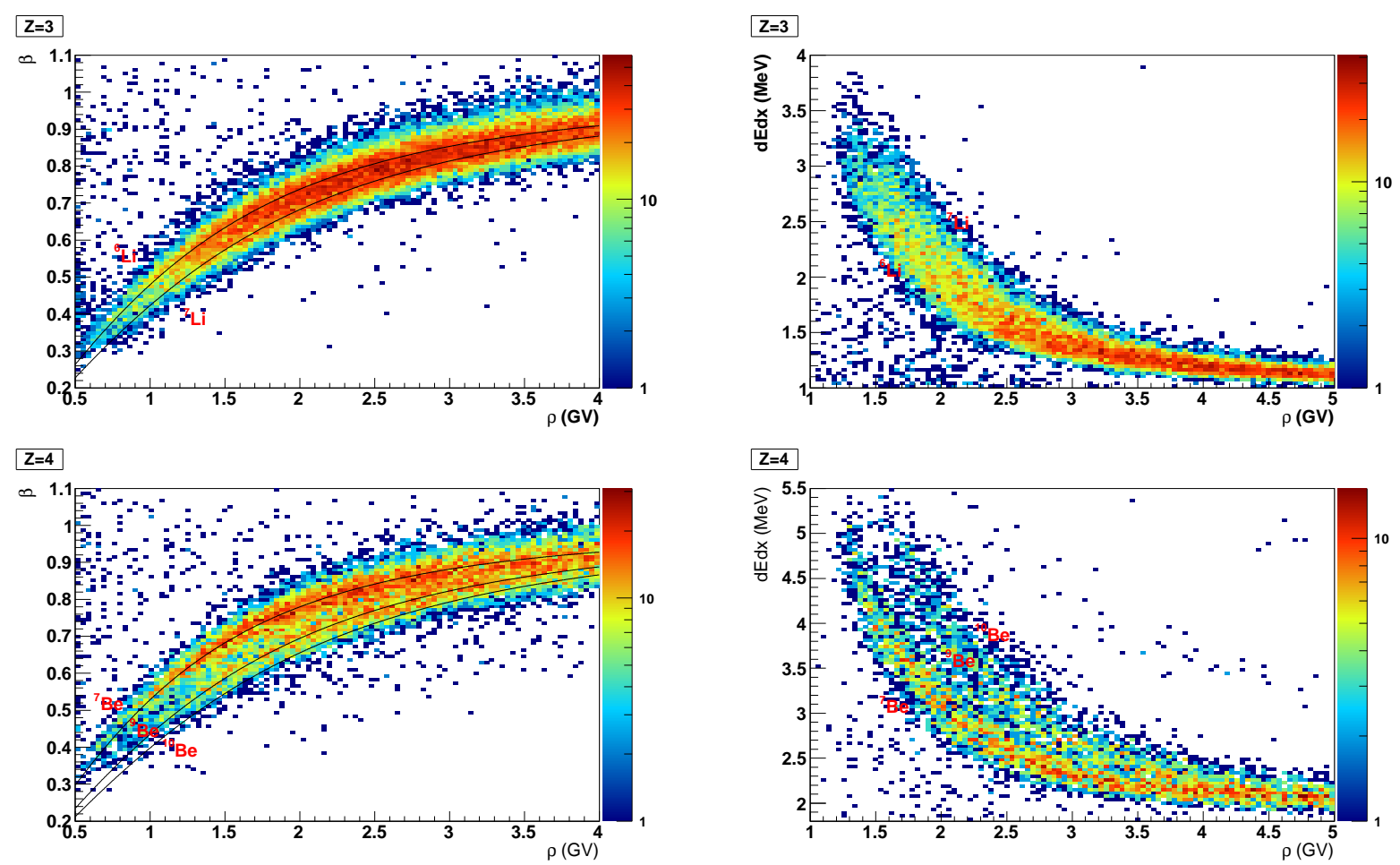

Figure 1: $\beta$ vs. rigidity for $Z=3$ (top) and $Z=4$ Figure 2: Mass separation for $Z=3$ (top) and $Z=4$ (bottom) particles. The black lines represent the ex(bottom) particles using the "truncated mean"-method. pectations for each isotope.

loss distributions shows a Landau tail which degrades the resolution of the $\mathrm{dE} / \mathrm{dx}$ measurement. Using a truncation method, the $50 \%$ of samples with larger pulse amplitudes are excluded before taking the mean of the $\mathrm{dE} / \mathrm{dx}$ measurements, thus reducing the effect of the Landau tail. Similar to the selection in [B], an energy dependent lower limit on the remaining number of layers was used to get a high selection efficiency and a lower energy limit of the analysis of around $200-300 \mathrm{MeV} / \mathrm{n}$. In Fig. $\square$ the mean $d E / d x$ for each event vs. the rigidity for $Z=3$ and $Z=4$ particles is shown. The energy loss in $\mathrm{MeV}$ was derived from the measurement in MIP using a conversion factor. In both plots the isotopic separation is clearly visible.

\subsection{Mass resolution}

In a magnetic spectrometer similar to PAMELA the mass resolution depends on three independent contributions: The precision of the velocity measurement (given either by timing or by measuring the energy loss), the bending power of the magnetic spectrometer coupled with the intrinsic limits of spatial resolution which the tracking detectors provide $\left(M D R_{\text {spec }}\right)$, and the multiple scattering of the particle along its path in the bending area of the magnet $\left(M D R_{\text {cou }}\right)$. The $M D R_{\text {spec }}$ for $Z=3$ particles is about $1000 \mathrm{GV}$ while for $Z=4$ particles it is about $500 \mathrm{GV}$ (due to saturation of the silicon strips in the tracker which reduces the spatial resolution). In any case the contribution of $M D R_{\text {spec }}$ to the overall mass resolution is negligible for the particles we are analyzing (up to some GV), at low energies the contribution from multiple scattering is the dominant effect. Its 
value is inverse proportional to the bending power of the magnet $\left(\int B \cdot d l\right)$ and direct proportional to the amount of matter traversed along the bending part of the track. For the PAMELA spectrometer we found a value for $M D R_{\text {соu }}$ around $3.5 \%$ at $8 \mathrm{GV}$, increasing to about $6 \%$ at $1 \mathrm{GV}$ [四], which sets the lower limit for the mass resolution, the contribution of the velocity measurement has then to be added to get the overall value. The mass resolution for simulated and flight data was analyzed in detail and was found to agree quite well, however, due to dominant effect of multiple scatter, the best resolution one can achieve is around 6\%, thus giving for example about 0.36 amu for ${ }^{6} \mathrm{Li}$ particles. For more details see [Q].

\subsection{Raw isotope numbers with the ToF and calorimeter}

We have two complementing experimental methods to separate the isotopes: The combination of magnetic spectrometer either with the TOF or with the multiple $d E / d x$ measurements within the calorimeter. The isotope separation as well as the determination of isotope ratios was performed identical to [3] in intervals of kinetic energy per nucleon. Since the magnetic spectrometer measures the rigidity of particles, this implies different rigidity intervals according to mass of the the isotope under study. The $d E / d x$ distributions of the calorimeter have a non-gaussian shape, therefore one has to model the expected distributions of the observable quantities and then perform likelihood fits, we used the "TFractionFitter" toolkit for this task. First one has to create the expected $d E / d x$ distributions for each isotope, this is done using the full Monte Carlo simulation of the PAMELA apparatus based on the GEANT 4 code, which has been already described [ $Q$ ], [3]. The $1 / \beta$ distributions of the ToF have a gaussian shape and could be fitted with gaussian functions, as it is done in the analysis of the hydrogen and helium isotopes ([B]], however, in this work we used the same approach as for the calorimeter and created $1 / \beta$ distributions using the simulation, and then used "TFractionFitter" for the Likelihood analysis.

As an example we show in Fig. [1] the distributions ToF and calorimeter for lithium in the 2.1 $2.3 \mathrm{GV}$ rigidity range, and in Fig. (t similar distributions for beryllium. The grey area shows how the combined fit using the two (for lithium) or three (for beryllium) distributions derived with the modified GEANT 4 simulation matches the data points (black points) while the colored areas shows the estimated individual isotope signals.

\subsection{Systematic Error of the Likelihood Fits}

When analyzing hydrogen and helium isotopes with the calorimeter, taking the simulated energy loss in each layer as coming from GEANT 4, we noticed that the resulting distributions showed a slight mismatch from the flight data. We found that the width of the histograms was smaller than in the real data, also there was a small offset. We applied a multiplicative factor to the simulated energy loss in a layer, plus adding a gaussian spread of the signal of some percent. Also for the $1 / \beta$ distributions of the ToF small deviations were found, which is understandable, since the creation of the timing signals in our simulation is somewhat simplified (for example no light tracing in the scintillators) compared to the real physical processes. In the hydrogen and helium analysis this fine tuning of the simulated distributions was relatively easy, since the mass resolution was better than $0.3 \mathrm{amu}$ for these particles [B]], which makes it possible to separate the peaks of two neighbored isotopes. Also the statistics are high, and one can always use the dominant peak in the distributions $\left({ }^{1} \mathrm{H}\right.$ and $\left.{ }^{4} \mathrm{He}\right)$ for comparisons of flight data and simulation. In the analysis of lithium and beryllium 

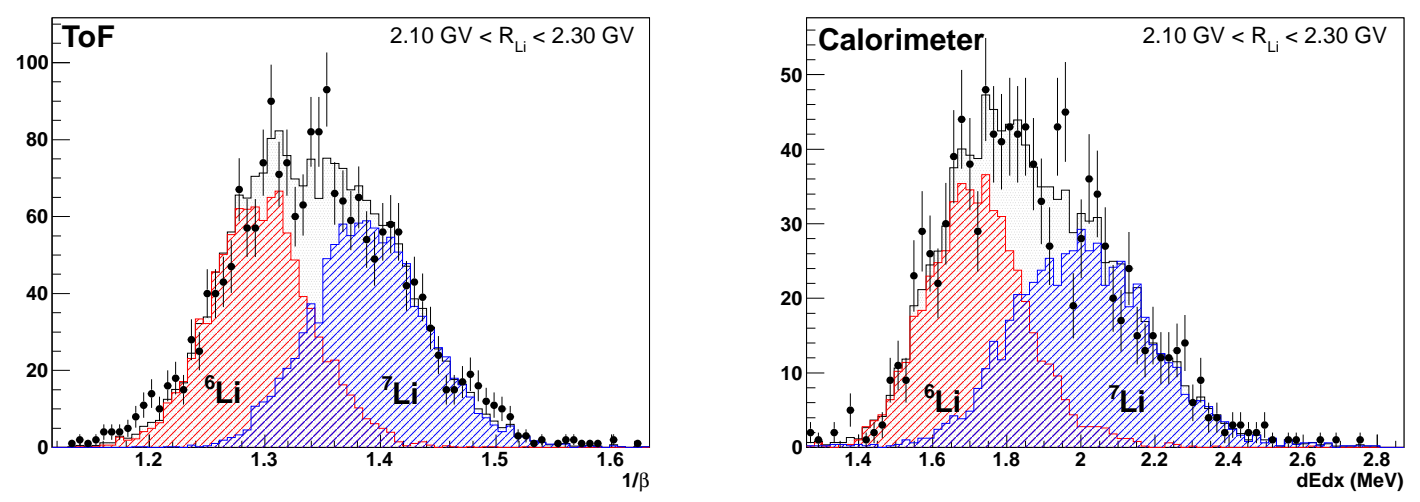

Figure 3: $1 / \beta$ distributions of the ToF (left) and truncated mean $d E / d x$ of the calorimeter (right) for lithium in the $2.1-2.3 \mathrm{GV}$ range.
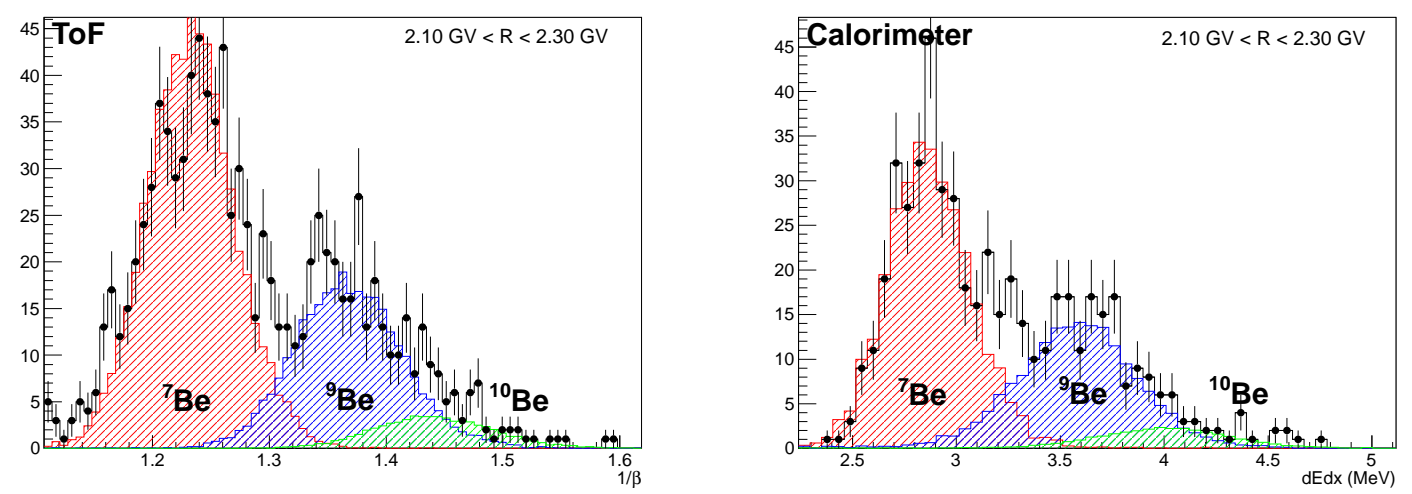

Figure 4: $1 / \beta$ distributions of the ToF (left) and truncated mean $d E / d x$ of the calorimeter (right) for beryllium in the $2.1-2.3 \mathrm{GV}$ range.

the mass resolution is $\geq 0.4 \mathrm{amu}$, thus a clear separation of the peaks of two neighbored isotopes is not possible, and the statistics is low. As one can see in Fig. 团, in the beryllium analysis the ${ }^{7} \mathrm{Be}$ peak can be clearly identified. We used the redundant detectors to separate a ${ }^{7} \mathrm{Be}$ sample from the flight data, and then compared these distributions for ToF or calorimeter with the simulation. As for hydrogen and helium we found a small rigidity-dependent offset, which is shown in Fig. 5 (left) for the ToF. We assigned a best-fit curve which is then used to correct the simulated data. ${ }^{6} \mathrm{Li}$ and ${ }^{7} \mathrm{Li}$ particles have almost the same abundance ( see Fig. B]), which makes it practically impossible to identify clear peaks in the flight data. Similar to the method used for beryllium, we used the redundant detectors to separate a ${ }^{6} \mathrm{Li}$ and ${ }^{7} \mathrm{Li}$ sample from the flight data, and then compared these distributions for ToF or calorimeter with the simulation. In Fig. [] (right) the offset for the calorimeter is shown. We assigned a mean best-fit curve to the ${ }^{6} \mathrm{Li}$ and ${ }^{7} \mathrm{Li}$ results and took the differences as a systematic error. We checked in detail how these offsets or different shapes of the simulated distributions contribute to the systematic error of the isotopic ratio. We found that the offsets give the major effect, a shape which is too "narrow" or too "wide" has a smaller effect. More details of this analysis will be given in a future paper. 

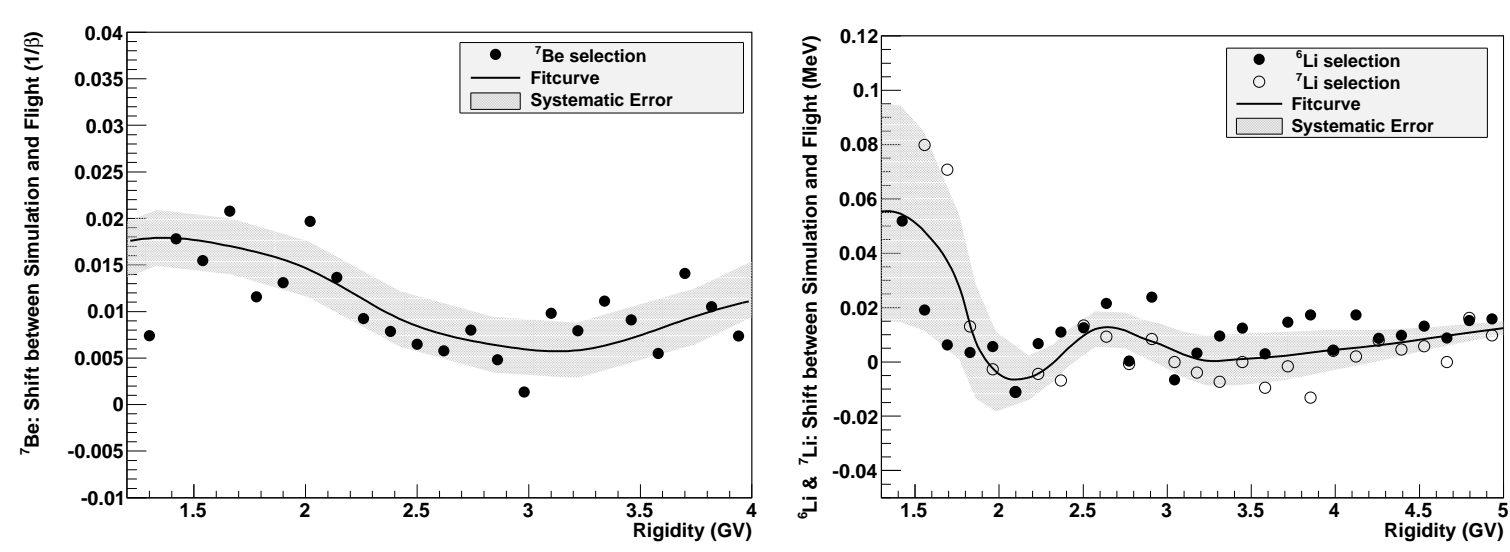

Figure 5: Difference between flight selected distributions and simulated distributions: ${ }^{7} \mathrm{Be}$ ToF $1 / \beta$ (left), ${ }^{6} \mathrm{Li}$ and ${ }^{7} \mathrm{Li}$ calorimeter $d E / d x$ (right)

\section{Isotopic ratios}

To derive the isotopic ratios, the numbers of selected events derived in the previous section using the likelihood fits have to be corrected for the selections efficiencies and particle losses. The efficiencies were mostly derived using simulations, these results were then checked using flight data at low energies, where the creation of test samples is possible using redundant detectors. Furthermore one needs the geometrical factor and the live time of the instrument as evaluated by the trigger system [B] , since these values depend on the rigidity. The finite resolution of the magnetic spectrometer and particle slowdown due to ionization energy losses results in a distortion of the particle spectra, which affects the isotopic ratio. We employed a Bayesian unfolding procedure to correct for this effect ([B] , [四).

\section{Results and discussion}

In the following we show some preliminary results for the ratios ${ }^{7} \mathrm{Li} /{ }^{6} \mathrm{Li}$ and ${ }^{7} \mathrm{Be} /\left({ }^{9} \mathrm{Be}+{ }^{10} \mathrm{Be}\right)$ derived with the ToF and the calorimeter together with other measurements. These preliminary results show a good agreement between the measurements with the ToF and the calorimeter, which gives confidence in the results. The main contribution to the systematic error of the ratios is the systematic uncertainty of the displacement of the simulated distributions used for the LikelihoodFit (see section B.5).

\section{Acknowledgement}

We acknowledge support from the Russian Space Agency (Roscosmos), the Russian Ministry of Education and Science, project №3.2131.2017, Deutsches Zentrum fur Luft- und Raumfahrt (DLR), the Swedish National Space Board, and the Swedish Research Council. The Italian authors acknowledge the partial financial support from The Italian Space Agency (ASI) under the program "Programma PAMELA - attivita' scientifica di analisi dati in fase E". 

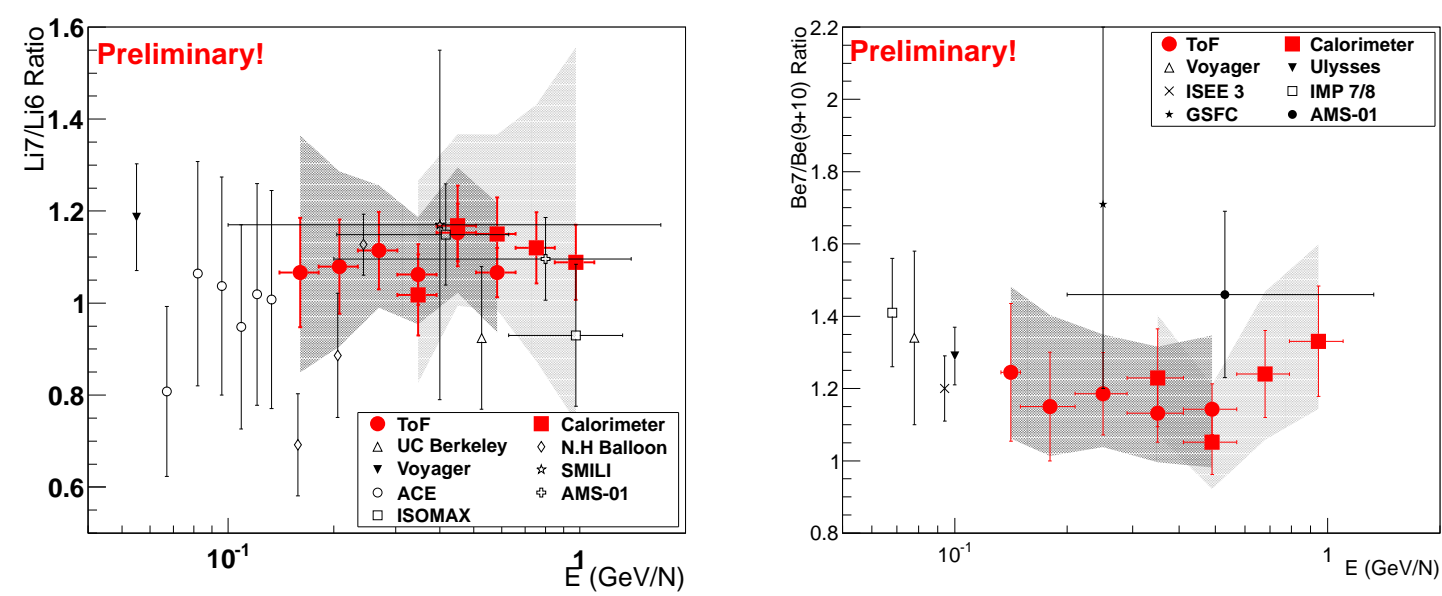

Figure 6: Results for the ratios ${ }^{7} \mathrm{Li} /{ }^{6} \mathrm{Li}$ and ${ }^{7} \mathrm{Be} /\left({ }^{9} \mathrm{Be}+{ }^{10} \mathrm{Be}\right)$ derived with PAMELA: ToF (red circles), calorimeter (red squares). Error bars show statistical uncertainty while the shaded area shows the systematic

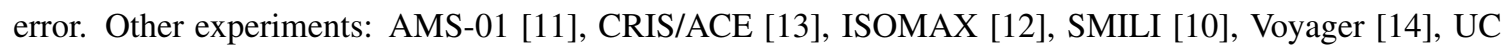

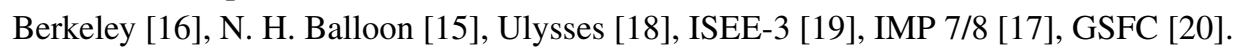

\section{References}

[1] O. Adriani et al. 2011, Science 33269 - Supplementary Online Material

[2] O. Adriani et al. 2014 Astrophys. J. 79130

[3] O. Adriani et al. 2016, Astrophys. J., 818, 1

[4] S. Agostinelli et al. 2003, Nucl. Instrum. Meth. A, 506, 250

[5] E. Anders and N. Grevesse 1989 Geochim. Cosmochim. Acta 53197

[6] M. Lemoine et al. 1993 Astron. Astrophys. 269469

[7] P. Picozza et al. 2007 Astropart. Phys. 27 296-315

[8] M. Boezio et al. 2002 Nucl. Instr. and Meth. A 487 407-422

[9] W. Menn et al. 2015 Proc. 34th Int. Cosmic Ray Conf. (The Hague) 269

[10] S. P. Ahlen et al. 2000 Astrophys. J. 534757

[11] M. Aguilar et al. 2011 Astrophys. J. 736105

[12] T. Hams et al. 2004 Astrophys. J. 611 892-905

[13] G. A. de Nolfo et al. 2006 Adv. Space Res. 38 1558-1564

[14] W. R. Webber, A. Lukasiak and F. B. McDonald 2002 Astrophys. J. 568 210-215

[15] W. R. Webber and J. Kish 1979 Proc. 16th Int. Cosmic Ray Conf. (Kyoto) 1389

[16] A. Buffington, C. D. Orth and T. S. Mast 1978 Astrophys. J. 226355

[17] M. Garcia-Munoz, G. M. Mason and J. A. Simpson 1977 Astrophys. J 217859

[18] J. J. Connell 1998 Astrophys. J. 501 L59

[19] M. E. Wiedenbeck and D. E. Greiner 1980 Astrophys. J. 239 L139

[20] F. A. Hagen, A. J. Fisher, and J. F. Ormes 1977 Astrophys. J. 212262 